\title{
Amplified charge and discharge rates in phase change materials for energy storage using spatially-enhanced thermal conductivity
}

\author{
Lien Chin Wei ${ }^{\mathrm{a}}$, Jonathan A. Malen ${ }^{\mathrm{a}, \mathrm{b}, *}$ \\ ${ }^{a}$ Department of Mechanical Engineering, Carnegie Mellon University, Pittsburgh, PA 15213, USA \\ ${ }^{b}$ Department of Materials Science and Engineering, Carnegie Mellon University, Pittsburgh, PA 15213, USA
}

\begin{abstract}
Composites made with high thermal conductivity meshes embedded in phase change materials (PCMs) increase charge/discharge rates of latent heat energy storage systems. We study the benefits of spatially-dependent enhancements to thermal conductivity on the charge/discharge rates of PCMs in both one-dimensional Cartesian and one-dimensional cylindrical coordinates. Our nondimensionalized quasi-steady (Stefan number $<\sim 0.1$ ) solution indicates that the average charge (discharge) rate in a spatially-enhanced PCM outperforms the uniformly-enhanced case by maximizing the enhancement near the heat source and therein reducing the time averaged thermal resistance to melting (solidifying). Relative to a uniformly-enhanced thermal conductivity, the optimal charge/discharge rate enhancement is a modest $12 \%$ in one-dimensional Cartesian coordinates but as high as $140 \%$ in one-dimensional cylindrical coordinates. Our analytical solutions are a design guide for graded mesh structures that can be realized by advanced fabrication techniques such as additive manufacturing and applied in applications ranging from telecommunications to buildings, where PCMs are employed to harness rapidly varying energy sources.
\end{abstract}

Keywords: Stefan problem; thermal conductivity; phase change material; effective medium; additive manufacturing; latent heat

\section{Introduction}

The present day mismatch between increasing power demand and limited energy resources motivates the discovery of novel and renewable energy sources and improvements to the energy efficiency of power conversion systems [13]. To improve utilization of renewable resources, energy storage devices and systems that reduce the imbalance between intermittent supply and demand are critical [2]. Research on thermal energy storage has improved the design

\footnotetext{
*Corresponding author

Email address: jonmalen@andrew.cmu.edu (Jonathan A. Malen)

Preprint submitted to Applied Energy
}

of green buildings [4, 5], utilization of solar thermal energy [6-8], and recovery of waste heat [1].

Thermal energy can be stored in the form of sensible heat and latent heat [1]. Thermal energy storage in the form of sensible heat occurs primarily through the activation of vibrations, realized as heat capacity within the storage material. Thermal energy storage in the form of latent heat occurs through the breaking of chemical bonds when a substance changes from one phase to another phase at a constant temperature. Phase change materials (PCMs) can store more energy per unit volume in the form of latent heat than can be stored by sensible heat at practical 


\begin{tabular}{|c|c|c|c|}
\hline \multicolumn{2}{|c|}{ Nomenclature } & \multicolumn{2}{|c|}{ Greek Symbol } \\
\hline & & $\epsilon$ & $\begin{array}{l}\text { enhancement ratio of the average charge/discharge } \\
\text { rate }\end{array}$ \\
\hline$\Delta \epsilon$ & relative difference of enhancement ratio & $\epsilon_{\mathrm{R} 2}$ & coefficient 2 in Eq. (21), $R_{\max }^{2}-1$ \\
\hline$\Delta k$ & thermal conductivity difference $\left(\mathrm{W} \cdot \mathrm{m}^{-1} \cdot \mathrm{K}^{-1}\right)$ & $\epsilon_{\mathrm{R} 3}$ & coefficient 3 in Eq. (21), $2 R_{\max }^{3}-3 R_{\max }^{2}+1$ \\
\hline$\Delta I$ & temperature anterence $(\mathrm{n})$ & $\kappa$ & dimensionless thermal conductivity \\
\hline$A$ & slope of $\kappa(\zeta)$ & $\omega$ & 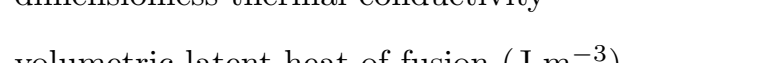 \\
\hline$B$ & intercept of $\kappa(\zeta)$ & $\lambda$ & vorumetric ratent neat or lusion $(\mathrm{J} \cdot \mathrm{m}$ ) \\
\hline$C_{\mathrm{p}}$ & volumetric heat capacity $\left(\mathrm{J} \cdot \mathrm{K}^{-1} \cdot \mathrm{m}^{-3}\right)$ & $\rho$ & $\begin{array}{l}\text { dimensionless radial position in cylindrical coor- } \\
\text { dinates }\end{array}$ \\
\hline$D$ & dimensionless diffusivity & $\rho_{\mathrm{m}}$ & dimensionless melting front position in cylindri- \\
\hline$d$ & length of PCM/mesh composites (m) & & cal coordinates \\
\hline$G$ & slope of $\kappa(\rho)$ & $\tau$ & dimensionless time \\
\hline$H$ & intercept of $\kappa(\rho)$ & $\theta$ & dimensionless temperature \\
\hline$k$ & thermal conductivity $\left(\mathrm{W} \cdot \mathrm{m}^{-1} \cdot \mathrm{K}^{-1}\right)$ & $\zeta$ & $\begin{array}{l}\text { dimensionless z position in Cartesian coordi- } \\
\text { nates }\end{array}$ \\
\hline$r$ & radial position in cylindrical coordinates (m) & & melting front nocition in Cortecion \\
\hline$r_{1}$ & inner radius of the $\mathrm{PCM} /$ mesh composites $(\mathrm{m})$ & $\zeta_{\mathrm{m}}$ & $\begin{array}{l}\text { amensionless melting iront position in cartesian } \\
\text { coordinates }\end{array}$ \\
\hline$r_{2}$ & outer radius of the $\mathrm{PCM} /$ mesh composites $(\mathrm{m})$ & Subsc & ript \\
\hline$R_{\max }$ & ratio of radii, $r_{2} / r_{1}$ & $r$ & 1-D cylindrical coordinates \\
\hline$R_{\min }$ & $R_{\max }-1$ & $\mathrm{~m}$ & melting front \\
\hline$R_{\mathrm{m}}$ & $r_{\mathrm{m}} / r_{1}=\rho_{\mathrm{m}}\left(R_{\min }\right)+1$ & $\max$ & maximum \\
\hline$r_{\mathrm{m}}$ & $\begin{array}{l}\text { melting front radial position in cylindrical coor- } \\
\text { dinates }(\mathrm{m})\end{array}$ & Mesh & metal mesh \\
\hline Ste & Stefan number & $\min$ & minimum \\
\hline$T$ & temperature $(\mathrm{K})$ & opt & optimal value \\
\hline$t$ & time $(\mathrm{s})$ & $\mathrm{PCM}$ & phase change material \\
\hline$v$ & volume fraction & $\mathrm{s}$ & surface \\
\hline$z$ & $\mathrm{z}$ position in Cartesian coordinates $(\mathrm{m})$ & SE & spatial enhancement \\
\hline$z_{\mathrm{m}}$ & melting front position in Cartesian coordinates & UE & uniform enhancement \\
\hline & $(\mathrm{m})$ & $\mathrm{z}$ & 1-D Cartesian coordinates \\
\hline
\end{tabular}

temperature differences. For example, the temperature of a wax such as n-Octadecane (latent heat: $243.5 \mathrm{~kJ} \cdot \mathrm{kg}^{-1}$ at a melting temperature of $300.8 \mathrm{~K}$ and solid phase specific heat: $2.14 \mathrm{~kJ} \cdot \mathrm{kg}^{-1} \cdot \mathrm{K}^{-1}$ [9]) has to be increased by more than $110 \mathrm{~K}$ for the sensible heat to equal the latent heat of fusion. Moreover, extended storage of energy at this elevated temperature requires increased insulation relative to the PCM, which can be stored just above its melting temperature therein limiting the temperature difference driving heat loss. Hence, energy storage systems incorpo- 
rating PCMs are used to meet high energy storage requirements in applications such as solar thermal power plants and thermal management systems $[7,10]$. PCM-filled heat exchangers that rapidly dissipate heat can be employed for thermal management in small-sized dynamic systems, such as telecommunications devices and home appliances [11]. PCM-embedded (paraffin/expanded graphite) wall boards that react quickly to regulate temperature can reduce and shift the average consumption and peak loading of building electricity demands [12].

Energy storage rates (also known as charge rates) of PCMs are governed by their thermal conductivity, which dictates the rate that heat reaches the solid-liquid interface. Low thermal conductivities of PCMs limit the charge (discharge) rate during melting (solidification) [13]. High thermal conductivity fillers such as carbon fiber, carbon nanotubes, graphite, graphite flakes, graphene nanocomposites, and expanded graphite have been dispersed into PCMs to increase their thermal conductivities [14-19]. However, the dispersion of these particle-like fillers may not be stable after long-term usage [20]. Nanoscale dispersed fillers also encounter appreciable thermal interface resistance between the filler and the PCM that limits their effectiveness [21].

Another method to overcome the low thermal conductivity of PCMs is through the incorporation of a high thermal conductivity porous structure [22] (e.g., foams or metal meshes) as depicted in Fig. 1a. These structures are fixed, non-moving and continuous [23]. Successful implementation of high thermal conductivity porous structures made of copper, aluminum, and graphite have been investigated [24-27]. Non-metals, such as PCM-expanded graphite were also shown to experimentally shorten charging times [26]. For ultrathin-graphite foams (UGF), Ji et al. [21] found that the enhancement in thermal conductivity per unit volume fraction exceeded metal fillers. They [21] also predicted the composite's thermal conductivity using a specific rule of mixtures [28] based on the structure and the volume fraction of UGF. In all of these cases, the enhancement of thermal conductivity was spatially uniform because the porous matrices were homogeneous.

Although the uniformly-enhanced thermal conductivity in a mesh embedded PCM composite in Fig. 1a enhances the charge/discharge rates, we analyze whether a spatiallyvaried thermal conductivity, depicted in Fig. 1b, can further enhance the charge/discharge rates while conserving the amount of added material. Due to the development of advanced fabrication techniques such as additive manufacturing (AM), spatially complex architectures can be readily fabricated, and a spatially-varied thermal conductivity is feasible $[29,30]$. The goal of our analysis is to determine the optimal spatially-varied thermal conductivity to charge/discharge PCMs faster than those with a uniformly-enhanced thermal conductivity. To do so, we develop novel analytical solutions to the one-dimensional (1-D) Stefan problem under the quasi-steady state approximation incorporating a spatially-varied thermal conductivity generalized as a linear function of space in 1-D Cartesian and 1-D cylindrical coordinates. All the equations and solutions are nondimensionalized in space and time and compared to those of the uniformly-enhanced case. We find that relative to uniform enhancement with identical total mass, the maximum enhancement is just $12 \%$ in the planar geometry but can be more than $100 \%$ in the cylindrical geometry.

Melting phenomena of PCMs involving heat conduction with phase change at a moving interface or melting front, 
(a)

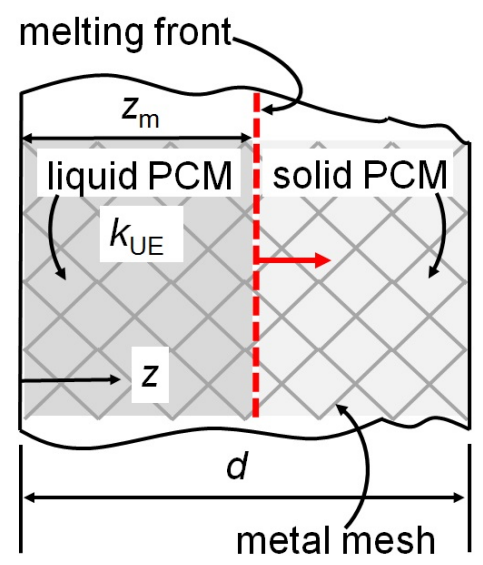

(b)

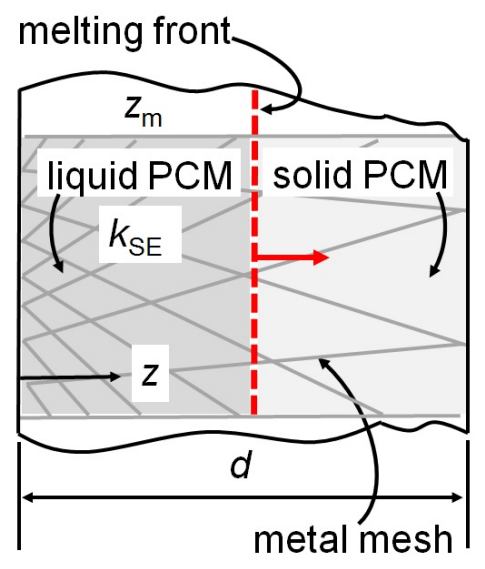

Figure 1: Conceptual schematics of metal meshes embedded in PCMs of length $d$ in 1-D Cartesian coordinates. (a) The volume fraction of metal mesh (gray lines) is uniform in space ( $z$ direction) and results in a uniform enhancement to thermal conductivity, $k_{\mathrm{UE}}$. (b) The volume fraction of metal mesh (gray lines) is a function of space. In this case, the mesh is most dense at $z=0$ and results in a spatially-enhanced thermal conductivity, $k_{\mathrm{SE}}=k(z)$ that is highest at $z=0$.

known as Stefan problems, were first studied in a onedimensional water-ice system [31]. Neumann used an error function to propose a classic solution [32]. Tarzia considered new problems with a moving interface and assembled explicit and approximate solutions involving heat and mass transfer [33]. An exponential distribution of mass diffusivity was recently considered to determine the moisture content in soybeans [34]. Similarity solutions using varying diffusivity as a function of temperature or position were also developed [35-41]. In Ref. [37], an alternative nonlinear thermal diffusivity distribution was considered for evaporation modeling in soil mechanics. This solution was later modified so the nonlinear term was thermal conductivity instead of thermal diffusivity [36, 37], and then further modified to include convective effects [38, 39]. Voller and Falcini [40] utilized a power law diffusivity in Cartesian coordinates, motivated by sedimentary mass transport scenarios in fluvial systems. We herein generalize Voller and Falcini's result by including parameters that independently control the intercept and slope of a linear distribution for thermal conductivity, and optimize them based on maximizing charge/discharge rates in PCMs.

\section{Methodology}

\subsection{The Stefan problem in Cartesian coordinates}

The one dimensional heat diffusion equation in a Stefan problem can be solved using the quasi-steady state approximation when the Stefan number Ste $<0.1$. The Stefan number is defined as the ratio of the sensible heat to the latent heat $S t e=C_{\mathrm{p}} \Delta T / \lambda$ where $C_{\mathrm{p}}$ is the volumetric specific heat of a PCM, $\Delta T=T_{\mathrm{s}}-T_{\mathrm{m}}$ where $T_{\mathrm{s}}$ is a temperature of a heat source on the surface and $T_{\mathrm{m}}$ is a melting temperature of the PCM, and $\lambda$ is the volumetric latent heat of fusion for the PCM [32]. The quasi-steady heat diffusion equation is expressed as

$$
\frac{\partial}{\partial z}\left[k(z) \frac{\partial T(z, t)}{\partial z}\right]=0
$$

where $\mathrm{z}$ is the coordinate, $k(z)$ is the spatially-varied thermal conductivity of a composite, $T(z, t)$ is the temperature of the PCM, and $t$ is time. Schematics of PCM composites of length $d$ with uniformly-enhanced thermal conductivity $k_{\mathrm{UE}}$ and spatially-enhanced thermal conductivity $k_{\mathrm{SE}}$ are shown in Fig. 1a and Fig. 1b. The volume fraction of 
metal mesh in Fig. 1a is uniform in space and results in $k_{\mathrm{UE}}$, while the volume fraction of metal mesh in Fig. $1 \mathrm{~b}$ is most dense at $z=0$ and results in $k_{\mathrm{SE}}=k(z)$ that is highest at $z=0$.

With constant temperature boundary conditions of $T(0, t)=T_{\mathrm{s}}$ and $T\left(z_{\mathrm{m}}(t), t\right)=T_{\mathrm{m}}$, the temperature of the composite for $0 \leq z \leq z_{\mathrm{m}}$ is solved, where $z_{\mathrm{m}}$ is the time-dependent melting front position. The temperature in the solid phase (for a melt process) is assumed to be at $T_{\mathrm{m}}$, so no sensible heat is required.

The energy balance at the melting front [32], is,

$$
-\left.k(z) \frac{\partial T(z, t)}{\partial z}\right|_{z=z_{\mathrm{m}}(t)}=\lambda \frac{d z_{\mathrm{m}}(t)}{d t}
$$

For small mesh volume fractions it is reasonable to assume $\lambda$ is independent of $z$ though this is indeed an approximation made to facilitate analytical solutions (in Appendix A we validate this assumption for volume fractions less than 0.2.). The initial condition for the melting front position $z_{\mathrm{m}}$ in Eq. (2) is $z_{\mathrm{m}}(t=0)=0$.

To nondimensionalize our result the thermal conductivity $k(z)$ in Eq. (1) is written as $k(\zeta)$ where a dimensionless coordinate is defined as $\zeta=z / d$, and $d$ is the length of the PCM/mesh composites shown in Fig. 1 ( $\zeta$ ranges from 0 to 1.$)$. Then, we define $\kappa(\zeta)=k(\zeta) / k_{\mathrm{UE}}$ and assume a linear form of $\kappa(\zeta)$ in Cartesian coordinates as

$$
\kappa(\zeta)=A \zeta+B
$$

where $A$ and $B$ are adjustable coefficients. This linear form of $\kappa(\zeta)$, where the slope and intercept can be independently varied, was chosen for simplicity to reach analytical solutions. Ref. [36-40] also consider heterogeneous diffusivities, but with differing forms relevant to their application in fluvial and oceanic sedimentary phenomena. A power law based dimensionless diffusivity $D \propto \zeta^{\frac{2 n-1}{n}}$ where $0<n<1$ is considered in Ref. [40]. Although $n=1$ creates a spatially-linear distribution, the intercept and the slope cannot be adjusted separately, which we later identify as crucial to enhancing PCM charge and discharge rates. Using our linear form provides straightforward proof that spatial variation of thermal conductivity can outperform the uniform PCM/mesh composite.

\subsection{Nondimensionalized solution for a spatially-enhanced thermal conductivity in 1-D Cartesian coordinates}

We further nondimensionalize Eq. (1) by substituting the dimensionless coordinate $\zeta=z / d$, thermal conductivity $\kappa(\zeta)=k(\zeta) / k_{\mathrm{UE}}$, and temperature $\theta\left(\zeta, \tau_{z}\right)=$ $\left[T(z, t)-T_{\mathrm{m}}\right] /\left(T_{\mathrm{s}}-T_{\mathrm{m}}\right)$ where $\tau_{z}=t / t_{z, \max }$ is the dimensionless time based upon $t_{z, \max }=\lambda d^{2} /\left[2 k_{\mathrm{UE}}\left(T_{\mathrm{s}}-T_{\mathrm{m}}\right)\right]$, the total melting time for a uniform $\mathrm{PCM} / \mathrm{mesh}$ composite (see Appendix B for the derivation of $t_{z, \max }$ ). The dimensionless temperature ranges from 0 and 1 since the temperature difference between heat source $\left(T_{\mathrm{S}}\right)$ and melting front $\left(T_{\mathrm{m}}\right)$ is the maximum temperature difference during the melting process. Notably, if $\kappa(\zeta)=1(A=0, B=1)$, we recover the solution for $k_{\mathrm{UE}}$. By doing so, we arrive at the dimensionless heat diffusion equation for 1-D Cartesian coordinates

$$
\frac{\partial}{\partial \zeta}\left[\kappa(\zeta) \frac{\partial \theta\left(\zeta, \tau_{z}\right)}{\partial \zeta}\right]=0
$$

The dimensionless temperature distribution in the melted PCM from Eq. (4) using dimensionless boundary conditions $\left(\theta=1\right.$ at $\zeta=0$, and $\theta=0$ at $\left.\zeta=\zeta_{\mathrm{m}}=z_{\mathrm{m}} / d\right)$ is obtained as 


$$
\theta\left(\zeta, \tau_{z}\right)=1-\frac{\ln \left(\frac{A \zeta}{B}+1\right)}{\ln \left(\frac{A \zeta_{\mathrm{m}}}{B}+1\right)}
$$

The energy balance from Eq. (2) can be similarly nondimensionalized as

$$
-\left.\kappa(\zeta) \frac{\partial \theta\left(\zeta, \tau_{z}\right)}{\partial \zeta}\right|_{\zeta=\zeta_{\mathrm{m}}\left(\tau_{z}\right)}=2 \frac{d \zeta_{\mathrm{m}}\left(\tau_{z}\right)}{d \tau_{z}}
$$

Solving Eq. (6) with the initial condition of $\zeta_{\mathrm{m}}\left(\tau_{z}=0\right)=0$ results in the following relationship between $\zeta_{\mathrm{m}}$ and $\tau_{z}$

$$
\tau_{z}=\frac{2}{A}\left[\ln \left(\frac{A \zeta_{m}}{B}+1\right)\left(\zeta_{m}+\frac{B}{A}\right)-\zeta_{\mathrm{m}}\right]
$$

Because the same amount of PCM is melted in both cases, $\tau_{z, \max }\left(\zeta_{\mathrm{m}}=1\right)$ is the total melting time of the spatially-enhanced PCM relative to the uniformlyenhanced PCM. A value of $\tau_{z, \max }$ less than one implies that a faster melting process takes place. Hence, the average charge rate is proportional to $1 / \tau_{z, \max }$. The enhancement ratio $\epsilon_{z}$ of the average charge rate of the spatiallyenhanced PCM to that of the uniformly-enhanced PCM is hence equivalent to the reciprocal of Eq. (7) with $\zeta_{\mathrm{m}}=1$

$$
\epsilon_{z}(A, B)=\frac{A}{2\left[\ln \left(\frac{A}{B}+1\right)\left(1+\frac{B}{A}\right)-1\right]}
$$

\subsection{Validation of quasi-steady state solution in 1-D Cartesian coordinates}

Our quasi-steady solution is compared with an exact similarity solution using a spatially-dependent thermal diffusivity [40].
In Ref. [40], the diffusivity is formulated as a power law of position, $\zeta^{\frac{2 n-1}{n}}$ where $0<n<1$. In Fig. 2, we compare our results to their solutions with $n=0.5$ and $n \simeq 1$. For a constant diffusivity $(n=0.5)$ in Fig. 2 , we set $\kappa=1$ in Eq. (3). For a nearly linear distribution with $n \simeq 1$, we use a spatially-dependent thermal conductivity $\kappa=A \zeta+B \simeq$ $\zeta$ with $A=1$ and $B \simeq 0$. The spatial dependence of $\kappa$ is shown in the inset of Fig. 2.

A comparison of the dimensionless position of the melting front $\zeta_{\mathrm{m}}$ is shown in Fig. 2. With $S t e=0.1$ the positions of the melting front under the quasi-steady state approximation with both constant thermal conductivity and spatially-dependent thermal conductivity are identical to Ref. [40].

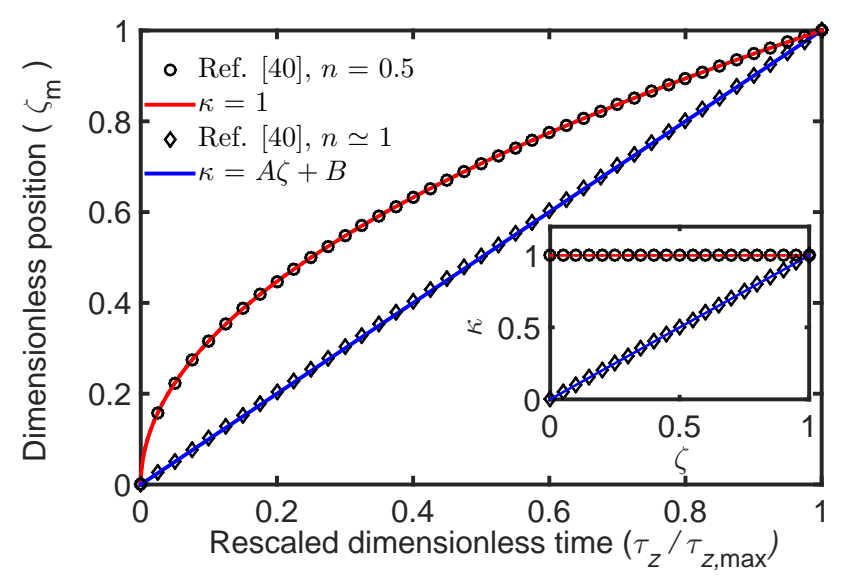

Figure 2: Dimensionless position of melting front vs. rescaled dimensionless time. The Stefan number of these similarity solutions is 0.1 . The diffusivity, which is proportional to $\zeta^{(2 n-1 / n)}$ with $n=0.5$ (Ref. [40], constant diffusivity) is approximated using Eq. (3) with $\kappa=1$. The nearly linear distribution with $n \simeq 1$ in Ref. [40] is approximated using $\kappa=A \zeta+B \simeq \zeta$. Under these conditions, the positions of the dimensionless melting front predicted by the quasisteady state approximations match these exact solutions.

\subsection{The Stefan problem in 1-D cylindrical coordinates}

Cylindrical systems that experience temperature gradients in the radial direction are prevalent in energy storage applications [42]. During the melting process, the temperature $T\left(r_{1}, t\right)=T_{s}$ at the inner surface $r=r_{1}$ is higher than the melting temperature $T\left(r_{\mathrm{m}}, t\right)=T_{\mathrm{m}}$ at $r=r_{\mathrm{m}}$ in Fig. 3 where $r$ is coordinate, $r_{1}$ is the inner 

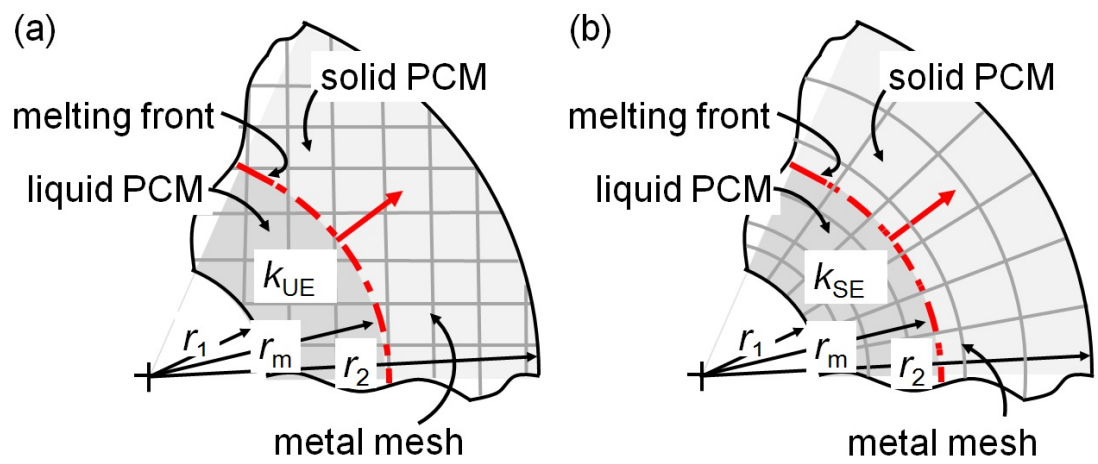

Figure 3: Conceptual schematics of a metal mesh of embedded in a PCM with inner radius $r_{1}$ and outer radius $r_{2}$ in the 1 -D cylindrical case. (a) The uniform metal mesh results in a uniform enhancement to thermal conductivity, $k_{\mathrm{UE}}$. (b) The graded metal mesh as a function of $r$ results in spatially-enhanced thermal conductivity, $k(r)$.

radius of the $\mathrm{PCM} /$ mesh composite, and $r_{\mathrm{m}}$ is the melting front position. The melting process starts at $r=r_{1}$ and progresses until the melting front arrives at the outer surface at $r=r_{2}$. The heat diffusion equation in a onedimensional cylindrical system is

$$
\frac{1}{r} \frac{\partial}{\partial r}\left[r k(r) \frac{\partial T(r, t)}{\partial r}\right]=0 .
$$

The temporal position of the melting front is again determined using the energy balance at $r=r_{\mathrm{m}}(t)$

$$
-\left.k(r) \frac{\partial T(r, t)}{\partial r}\right|_{r=r_{\mathrm{m}}(t)}=\lambda \frac{d r_{\mathrm{m}}(t)}{d t}
$$

The initial condition for the melting front is $r_{\mathrm{m}}(t=0)=$ $r_{1}$.

The radial coordinate is nondimensionalized by the thickness of the annulus $\left(r_{2}-r_{1}\right)$ of the PCM/mesh composite as shown in Fig. 3, and the dimensionless radial coordinate defined as $\rho=\left(r-r_{1}\right) /\left(r_{2}-r_{1}\right)$ ranges from 0 to 1 . Thus, the dimensionless thermal conductivity of linear radial dependence is

$$
\kappa(\rho)=G \rho+H
$$

where $G$ is the slope and $H$ is the intercept of the linear distribution.

\subsection{Nondimensionalized solution for a spatially-enhanced thermal conductivity in 1-D cylindrical coordinates}

Eq. (9) is nondimensionlized using the dimensionless radial coordinate $\rho=\left(r-r_{1}\right) /\left(r_{2}-r_{1}\right)$, ratio of radii $R_{\max }=r_{2} / r_{1}$, thermal conductivity $\kappa(\rho)=k(\rho) / k_{\mathrm{UE}}$, and temperature $\theta\left(\rho, \tau_{r}\right)=\left[T(r, t)-T_{\mathrm{m}}\right] /\left(T_{\mathrm{s}}-T_{\mathrm{m}}\right)$ where $\tau_{r}=t / t_{r, \max }$ is the dimensionless time based upon $t_{r, \max }=\lambda r_{1}^{2}\left[R_{\max }^{2} \ln \left(R_{\max }\right) / 2-R_{\max }^{2} / 4+1 / 4\right] /$ $\left[k_{\mathrm{UE}}\left(T_{\mathrm{S}}-T_{\mathrm{m}}\right)\right]$, the total melting time for a uniform $\mathrm{PCM} / \mathrm{mesh} /$ composite with $R_{\max }$ in $1-\mathrm{D}$ cylindrical coordinates (see Appendix $\mathrm{B}$ for the derivation of $t_{r, \max }$ ). The range of dimensionless radial coordinate and temperature are exactly the same as those in 1-D Cartesian coordinates, whereas the ratio of radii $R_{\max }=r_{2} / r_{1}$ quantifies the size of the PCM and mesh in terms of the relative radii of the annulus. By doing so, we arrive at the dimensionless heat diffusion equation for 1-D cylindrical coordinates 
$\frac{1}{\left[\rho+1 /\left(R_{\max }-1\right)\right]} \frac{\partial}{\partial \rho}\left[\left(\rho+\frac{1}{R_{\max }-1}\right) \kappa(\rho) \frac{\partial \theta\left(\rho, \tau_{r}\right)}{\partial \rho}\right]$ $=0$.

The dimensionless temperature distribution with boundary conditions $\left(\theta=1\right.$ at $\rho=0$ and $\theta=0$ at $\left.\rho=\rho_{\mathrm{m}}\right)$ is,

$$
\theta\left(\rho, \tau_{r}\right)=1-\frac{\ln \left(1+\rho R_{\min }\right)-\ln \left(\frac{G}{H} \rho+1\right)}{\ln \left(1+\rho_{\mathrm{m}} R_{\mathrm{min}}\right)-\ln \left(\frac{G}{H} \rho_{\mathrm{m}}+1\right)}
$$

where $\rho_{\mathrm{m}}=\rho_{\mathrm{m}}\left(\tau_{\mathrm{r}}\right)=\left(r_{\mathrm{m}}-r_{1}\right) /\left(r_{2}-r_{1}\right)$ is time dependent melting front position and $R_{\min }=R_{\max }-1$. The energy balance from Eq. (10) using all dimensionless terms in Eq. (12) and Eq. (13) (see Appendix C for the derivation of Eq. (14) and Eq. (15)) is

$$
\begin{aligned}
& -\left.\kappa(\rho) \frac{\partial \theta\left(\rho, \tau_{r}\right)}{\partial \rho}\right|_{\rho=\rho_{\mathrm{m}}\left(\tau_{r}\right)}= \\
& {\left[\frac{\left(R_{\max }-1\right)^{2}}{\frac{R_{\max }^{2}}{2} \ln \left(R_{\max }\right)-\frac{R_{\max }^{2}}{4}+\frac{1}{4}}\right] \frac{d \rho_{\mathrm{m}}\left(\tau_{r}\right)}{d \tau_{r}} .}
\end{aligned}
$$

As $R_{\max } \simeq 1$ (a thin cylindrical shell), the term in brackets approaches two, and Eq. (14) is the same as Eq. (6).

By solving Eq. (14) with the initial condition of $\rho_{\mathrm{m}}\left(\tau_{r}=\right.$ $0)=0$, the relationship between $\rho_{\mathrm{m}}$ and $\tau_{r}$ is

$$
\begin{aligned}
\tau_{r} & =\frac{R_{\min }}{\left(H R_{\min }-G\right)\left[\frac{R_{\max }^{2}}{2} \ln \left(R_{\max }\right)-\frac{R_{\max }^{2}}{4}+\frac{1}{4}\right]} \\
& \times\left[\frac{R_{\mathrm{m}}^{2}}{2} \ln \left(R_{\mathrm{m}}\right)+\frac{R_{\mathrm{m}}-1}{2}-\frac{H R_{\min }}{2 G}\left(R_{\mathrm{m}}-1\right)+\right. \\
& \left(-\frac{R_{\mathrm{m}}^{2}}{2}+\frac{H^{2} R_{\min }^{2}}{2 G^{2}}-\frac{H R_{\min }}{G}+\frac{1}{2}\right) \times \\
& \left.\ln \left(\frac{G}{H} \rho_{\mathrm{m}}+1\right)\right]
\end{aligned}
$$

where $R_{\mathrm{m}}=r_{\mathrm{m}} / r_{1}=\rho_{\mathrm{m}} R_{\min }+1$. When $r_{\mathrm{m}}=r_{2}$, then $R_{\mathrm{m}}=R_{\max }$ and $\rho_{\mathrm{m}}=1$ such that the enhancement ratio from the reciprocal of Eq. (15) is

$$
\begin{aligned}
& \epsilon_{r}\left(G, H, R_{\max }\right)= \\
& \frac{H R_{\min }-G}{R_{\min }}\left[\frac{R_{\max }^{2}}{2} \ln \left(R_{\max }\right)-\frac{R_{\max }^{2}}{4}+\frac{1}{4}\right] \\
& /\left[\frac{R_{\max }^{2}}{2} \ln \left(R_{\max }\right)+\frac{R_{\min }}{2}-\frac{H R_{\min }^{2}}{2 G}+\right. \\
& \left(-\frac{R_{\max }^{2}}{2}+\frac{H^{2} R_{\min }^{2}}{2 G^{2}}-\frac{H R_{\min }}{G}+\frac{1}{2}\right) \times \\
& \left.\ln \left(\frac{G}{H}+1\right)\right] .
\end{aligned}
$$

\subsection{Parameterization of thermal conductivity of a binary} composite

Next we apply restrictions that equate the average volume fraction of metal mesh in the UE and SE cases. We first need to relate the thermal conductivity of the composite to the volume fraction of mesh and PCM in the composite. Analytical effective medium models for the thermal conductivity of heterogeneous or composite materials described as functions of the component materials' volume fractions and thermal conductivities were reviewed by Wang et al. [43]. To predict the effective thermal conductivity of a binary mixture like a metal/PCM composite, five basic structural models (two forms of the Maxwell-Eucken, the Effective Medium Theory, the Series, and the Parallel model) based on the constituent volume fractions were generalized [43]. Based on the structure of metal/PCM composites where the metal mesh and PCM are bi-continuous phases, the Parallel Model (i.e., the rule of mixtures) is chosen to predict the effective thermal conductivity in a uniformly-enhanced case. This model defines $k_{\mathrm{UE}}=k_{\mathrm{Mesh}} v_{\mathrm{Mesh}}+k_{\mathrm{PCM}}\left(1-v_{\mathrm{Mesh}}\right)$ [43] where $k_{\text {Mesh }}$ is the thermal conductivity of the metal mesh, $v_{\text {Mesh }}$ 
is the volume fraction of the metal mesh, and $k_{\mathrm{PCM}}$ is the thermal conductivity of the PCM. As previously noted, a modified rule of mixtures based on the structure and volume fraction of graphite foams accurately predicted the thermal conductivity of UGF-erythritol composite [21]

Within the Parallel model, we modify $v_{\text {Mesh }}$ to be a function of position $v_{\text {Mesh }}^{\prime}(z)$ and $v_{\text {Mesh }}^{\prime}(r)$. For 1-D Cartesian coordinates, $k(z)=k_{\mathrm{Mesh}} v_{\mathrm{Mesh}}^{\prime}(z)+k_{\mathrm{PCM}}\left[1-v_{\mathrm{Mesh}}^{\prime}(z)\right]$. To create a fair comparison the average volume fraction of the metal mesh in the spatially-enhanced case is equated to that in the uniform case $v_{\mathrm{Mesh}}=\bar{v}_{\mathrm{Mesh}}^{\prime}=\int_{0}^{d} d z v_{\mathrm{Mesh}}^{\prime}(z) / d$. Under this constraint, $A=2(1-B)$, which makes the dimensionless thermal conductivity from Eq. (3) a function of only $B$

$$
\kappa(\zeta)=2(1-B) \zeta+B
$$

Therefore, the enhancement ratio $\epsilon_{z}$ in Eq. (8) takes the form

$$
\epsilon_{z}(B)=\frac{1-B}{\left[\ln \left(\frac{2(1-B)}{B}+1\right)\left(1+\frac{B}{2(1-B)}\right)-1\right]}
$$

Because $\kappa$ is linear in $\zeta$, the minimum $\kappa=k_{\mathrm{PCM}} / k_{\mathrm{UE}}=$ $\left[v_{\text {Mesh }}\left(k_{\text {Mesh }} / k_{\mathrm{PCM}}-1\right)+1\right]^{-1}$ occurs either at $\zeta=0$ or at $\zeta=1$, and the range of the intercept $B$ is

$$
\begin{aligned}
& {\left[v_{\text {Mesh }}\left(k_{\mathrm{Mesh}} / k_{\mathrm{PCM}}-1\right)+1\right]^{-1}<B<} \\
& 2-\left[v_{\mathrm{Mesh}}\left(k_{\mathrm{Mesh}} / k_{\mathrm{PCM}}-1\right)+1\right]^{-1} .
\end{aligned}
$$

As $k_{\mathrm{PCM}} / k_{\mathrm{Mesh}}$ approaches zero, the range of the intercept $B$ is $0<B<2$.

For a 1-D cylindrical coordinates, the spatially- enhanced thermal conductivity is written as $k(r)=k_{\mathrm{Mesh}} v_{\mathrm{Mesh}}^{\prime}(r)+k_{\mathrm{PCM}}\left[1-v_{\mathrm{Mesh}}^{\prime}(r)\right]$. Equating the average volume fraction, $v_{\text {Mesh }}=$ $\bar{v}_{\text {Mesh }}^{\prime}=\int_{r_{1}}^{r_{2}} 2 \pi r d r \quad v_{\text {Mesh }}^{\prime}(r) /\left(\pi r_{2}^{2}-\pi r_{1}^{2}\right)$, results in $G=\left[3(1-H)\left(R_{\max }+1\right) R_{\min }^{2}\right] /\left(2 R_{\max }^{3}-3 R_{\max }^{2}+1\right)$ in Eq. (11). Here, the dimensionless thermal conductivity as a function of $H$ and $R_{\max }$ is

$$
\kappa(\rho)=\frac{3(1-H)\left(R_{\max }+1\right) R_{\min }^{2}}{2 R_{\max }^{3}-3 R_{\max }^{2}+1} \rho+H .
$$

Thus, the enhancement ratio in Eq. (16) can be parameterized as

$$
\begin{aligned}
& \epsilon_{r}\left(H, R_{\max }\right)= \\
& {\left[H-\frac{3(1-H) \epsilon_{\mathrm{R} 2}}{\epsilon_{\mathrm{R} 3}}\right]\left[\frac{R_{\max }^{2}}{2} \ln \left(R_{\max }\right)-\frac{R_{\max }^{2}}{4}+\frac{1}{4}\right]} \\
& /\left\{\frac{R_{\max }^{2}}{2} \ln \left(R_{\max }\right)+\frac{R_{\min }}{2}-\frac{H \epsilon_{\mathrm{R} 3}}{6(1-H)\left(R_{\max }+1\right)}+\right. \\
& \left(\frac{1-R_{\max }^{2}}{2}\right) \ln \left[\frac{3(1-H)\left(R_{\max }+1\right) R_{\min }^{2}}{H \epsilon_{\mathrm{R} 3}}+1\right]+ \\
& {\left[\frac{H^{2} \epsilon_{\mathrm{R} 3}^{2}}{18(1-H)^{2} \epsilon_{\mathrm{R} 2}^{2}}-\frac{H \epsilon_{\mathrm{R} 3}}{3(1-H) \epsilon_{\mathrm{R} 2}}\right] \times} \\
& \left.\ln \left[\frac{3(1-H)\left(R_{\max }+1\right) R_{\min }^{2}}{H \epsilon_{\mathrm{R} 3}}+1\right]\right\},
\end{aligned}
$$

where $\epsilon_{\mathrm{R} 2}=R_{\max }^{2}-1$ and $\epsilon_{\mathrm{R} 3}=2 R_{\max }^{3}-3 R_{\max }^{2}+1$. The range of the intercept $H$ is

$$
\begin{aligned}
& \frac{1}{v_{\mathrm{Mesh}}\left(k_{\mathrm{Mesh}} / k_{\mathrm{PCM}}-1\right)+1}<H< \\
& \frac{3 R_{\max }^{3}-3 R_{\max }^{2}-3 R_{\max }+3}{R_{\max }^{3}-3 R_{\max }+2}- \\
& \frac{\epsilon_{\mathrm{R} 3}}{\left[v_{\mathrm{Mesh}}\left(k_{\mathrm{Mesh}} / k_{\mathrm{PCM}}-1\right)+1\right]\left(R_{\max }^{3}-3 R_{\max }+2\right)} .
\end{aligned}
$$

This range becomes $0<H<3$ when $k_{\mathrm{PCM}} / k_{\mathrm{Mesh}} \simeq 0$ and $1 / R_{\max } \simeq 0$. 


\section{Results and discussion}

The solutions from Eq. (18) and Eq. (21) are used to calculate the spatial distribution of $k$ that optimizes the average charge rate of the PCM composite.

\subsection{Spatial dependence of the melting front}

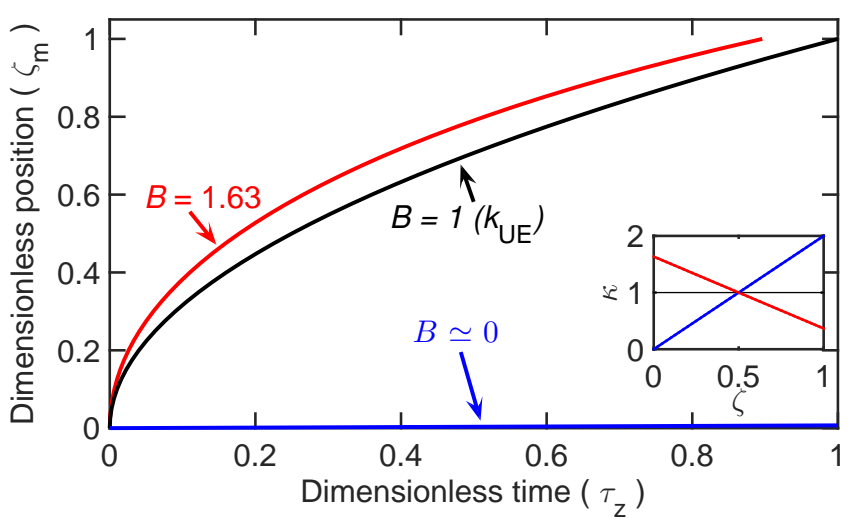

Figure 4: The melting front progresses differently with various intercepts $B$. The faster melting process with $B=1.63$ indicates higher average charge rate. The corresponding distributions of spatiallyenhanced thermal conductivity are shown in the inset where the heat source is at $\zeta=0$.

For 1-D Cartesian coordinates, the melting front positions as a function of time for three different values of $B$ are shown in Fig. 4. The corresponding distributions of $\kappa$ are shown in the inset of Fig. 4 . With $B \simeq 0$ the melting front progresses much slower than for $B=1\left(k_{\mathrm{UE}}\right)$. Notably the solution from [40] with $n=1$ is limited to this $B \simeq 0$ scenario. Alternatively, with $B=1.63$ the melting front reaches $\zeta_{\mathrm{m}}=1$ approximately $10 \%$ faster than with $B=1$ ( $B=1$ represents the uniformly-enhanced case). Notably the melting front with $B=1.63$ arrives at $\zeta_{\mathrm{m}}=0.5$ (half melted) $35 \%$ faster than the uniformlyenhanced case. Hence, by enhancing the thermal conductivity near to the heat source $(\zeta=0)$, the time averaged thermal resistance to get heat to the melting front is reduced, and the PCM can be charged/discharged faster.

\subsection{Optimization of mesh design}

The enhancement ratio $\epsilon_{z}$ is shown in Fig. 5 as a function of $B$ for 1-D Cartesian coordinates. We see that $\epsilon_{z}$ is enhanced between $B=1$ and $B=2\left(\epsilon_{z}>1\right)$, whereas, below $B=1$, the average heat transfer rate is worse than that with $k_{\mathrm{UE}}\left(\epsilon_{z}<1\right)$. These results can be used as a guide to design $k(z)$ that outperforms the uniformlyenhanced case.

The maximum $\epsilon_{z}$ occurs when the derivative $d \epsilon_{z}(B) / d B=0$ because the enhancement ratio as a function of $B$, shown in Fig. 5, is a concave-down curve. Fig. 5 indicates that $B=1.64$ is optimal and increases the charge rate over that of $k_{\mathrm{UE}}$ by $11.6 \%\left(\epsilon_{z, \mathrm{opt}}=1.116\right)$.

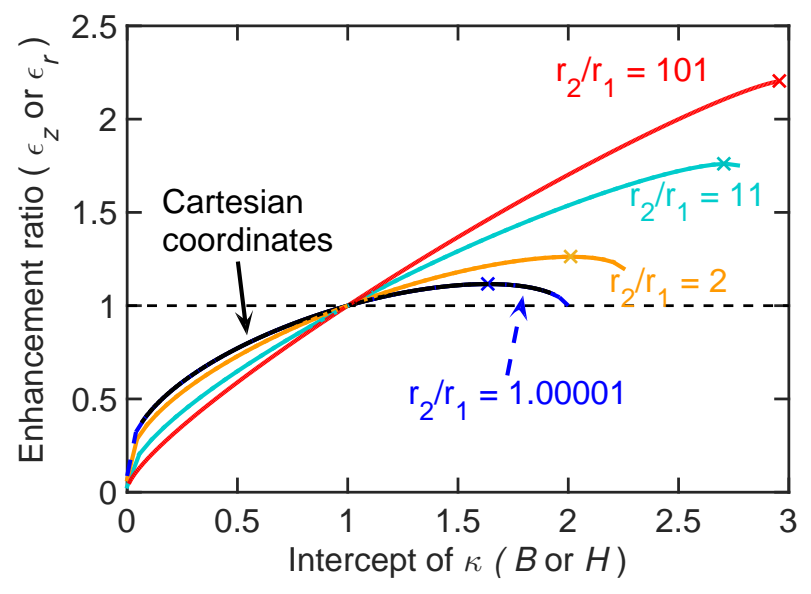

Figure 5: The enhancement ratio is a function of $B$ in Cartesian coordinates (Eq. (18)) and $H$ in 1-D cylindrical coordinates for several values of $r_{2} / r_{1}$ (Eq. (21)). The enhancement ratio in cylindrical coordinates with $r_{2} / r_{1}=1.00001$ is identical to that in a 1-D Cartesian coordinates. High radius ratios improve the average charge rate over that of uniform enhancement by more than $100 \%$. The range of $B$ and $H$ result from Eq. (19) and Eq. (22) using $v_{\text {Mesh }}=0.1$ and $k_{\mathrm{PCM}} / k_{\mathrm{Mesh}} \simeq 0.0007$.

Similarly, the enhancement ratio in 1-D cylindrical coordinates is shown in Fig. 5. The enhancement ratio $\epsilon_{r}$ in a cylindrical system varies with the radii ratio $r_{2} / r_{1}$ as well as the intercept $H$. First, we plot $\epsilon_{r}$ with $r_{2} / r_{1}=1.00001$ where the enhancement ratio is similar to that in 1-D Cartesian coordinates. This result confirms that the melting process in a thin cylindrical shell is approximated by 
the 1-D Cartesian coordinates solution. We further increase $r_{2} / r_{1}$ from 2 to 101 and plot $\epsilon_{r}$ in Fig. 5. The upper limit of $H$ approaches 3 and $\epsilon_{r}$ increases above 2 as $r_{2} / r_{1}$ increases. The crosses in Fig. 5 indicate the optimal enhancement ratio for each $r_{2} / r_{1}$.

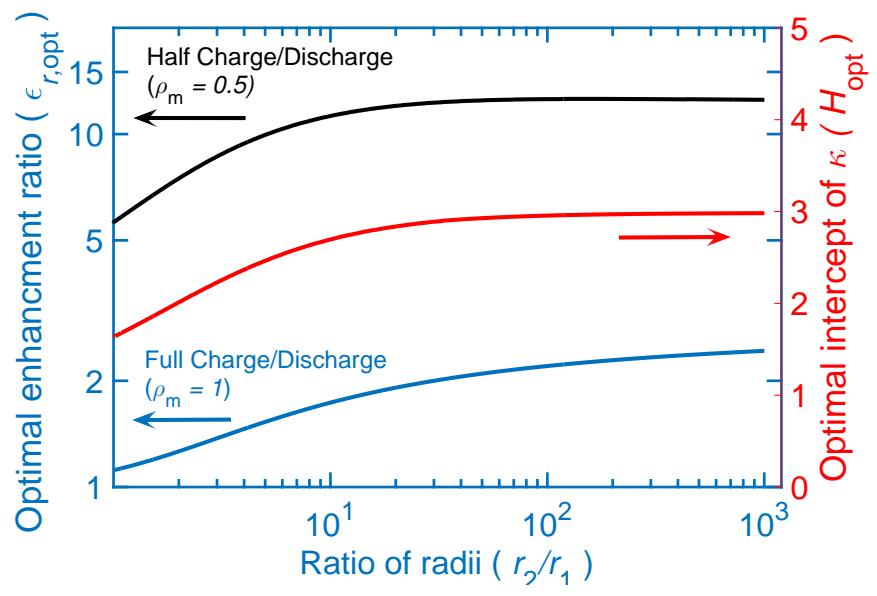

Figure 6: The optimal enhancement ratio $\epsilon_{r, \text { opt }}$ and optimal intercept $H_{\mathrm{opt}}$ as a function of the radius ratio $r_{2} / r_{1}$ at $\rho_{\mathrm{m}}=0.5$ and 1 . When the melting process is complete $\left(\rho_{\mathrm{m}}=1\right)$, the enhancement ratio ascends rapidly for $1<r_{2} / r_{1}<10$, then it gradually grows to 2.4 as $r_{2} / r_{1} \simeq 1000$. When the melting process is incomplete $\left(\rho_{\mathrm{m}}=0.5\right)$, even higher enhancement takes place.

The optimal enhancement ratio $\epsilon_{r, \text { opt }}$ and the corresponding intercept $H_{\mathrm{opt}}$ are shown in Fig. 6 as a function of $r_{2} / r_{1}$. As $r_{2} / r_{1} \simeq 1000, \epsilon_{r}$ approaches 2.4. More extreme enhancements can be achieved in cylindrical coordinates because in a homogeneous solid the derivative of thermal resistance, which represents the thermal resistance per unit cylindrical shell thickness, is highest at $r=r_{1}$ (i.e., thermal resistance accumulates most rapidly when the melt front is near $r=r_{1}$ ). As a result, spatial enhancement of thermal conductivity near the heat source at $r_{1}$ drastically reduces the time averaged thermal resistance during the charge/discharge process. It is remarkable that simply redistributing the mesh, while conserving its average volume fraction, results in enhancements to the charge/discharge rates exceeding $100 \%$ in the radial geometry system. Moreover, if the same meshes undergo an incomplete melting process such that $\rho_{\mathrm{m}}=0.5$, the average charge rate is enhanced even more than that of a complete melting processes $\left(\rho_{\mathrm{m}}=1\right)$, as shown in Fig. 6 .

\section{Conclusion}

We show that a spatially-enhanced thermal conductivity can enhance the charge/discharge rate in $\mathrm{PCM} / \mathrm{mesh}$ composites in both 1-D Cartesian and 1-D cylindrical coordinate systems. Our spatially-enhanced thermal conductivity uniquely incorporates a linear distribution with separately adjustable slope and intercept. Through nondimensionalizing the equations, we acquire and define the enhancement ratio of the average charge/discharge rates to that of the uniformly-enhanced case while conserving the average volume fraction of the metal mesh. We find that enhancing the thermal conductivity near the heat source effectively reduces the average thermal resistance throughout the phase change process, and thus improves the average charge rate by more than $100 \%$ in select cylindrical systems. Among other applications, enhanced charge/discharge rates will benefit thermal management in small dynamic systems and temperature regulation in buildings by PCMs. Likewise, equivalent performance can be achieved with less metal, which is appealing for applications that demand lightweight alternatives (e.g., a $\mathrm{Cu}$ mesh of volume fraction 0.1 nearly doubles the weight of a wax PCM.). Progress in advanced fabrication techniques such as additive manufacturing offers hope that complex metal meshes with spatially-varied thermal conductivity are a near-term reality $[29,30]$. Our work provides a critical guide for the design of such spatially-varied metal meshes. 


\section{Appendix A. Analysis adding spatially-dependent}

\section{volumetric latent heat}

As the volume fraction of a mesh $v_{\text {Mesh }}$ increases, the effective latent heat of fusion of the PCM composite decreases. For a uniformly-enhanced case, $\lambda$ in Eq. (2) can be modified as $\lambda_{\mathrm{UE}}=\lambda\left(1-v_{\mathrm{Mesh}}\right)$. For a spatially-enhanced case, $\lambda$ is replaced as $\lambda_{\mathrm{SE}}=\lambda\left(1-v_{\mathrm{Mesh}}^{\prime}\right)$. The dimensionless energy balance in 1-D Cartesian coordinates from Eq. (6) becomes

$$
\begin{aligned}
-\left.k(\zeta) \frac{\partial \theta\left(\zeta, \tau_{z}\right)}{\partial \zeta}\right|_{\zeta=\zeta_{\mathrm{m}}\left(\tau_{z}\right)} & =2 \frac{\lambda_{\mathrm{SE}}}{\lambda_{\mathrm{UE}}} \frac{d \zeta_{\mathrm{m}}}{d \tau_{z}} \\
& =2\left(\frac{1-v_{\mathrm{Mesh}}}{1-v_{\mathrm{Mesh}}^{\prime}}\right) \frac{d \zeta_{\mathrm{m}}}{d \tau_{z}}
\end{aligned}
$$

where $v_{\text {Mesh }}^{\prime}$ can be expressed using Eq. $\left(\kappa=k_{\mathrm{PCM}} / k_{\mathrm{UE}}+v_{\mathrm{Mesh}}^{\prime} \Delta k / k_{\mathrm{UE}} \quad\right.$ where $\Delta k=$ $k_{\text {Mesh }}-k_{\mathrm{PCM}}$, thermal conductivity difference) as a linear function

$$
v_{\mathrm{Mesh}}^{\prime}(\zeta)=\left[\frac{2 k_{\mathrm{UE}}(1-B)}{\Delta k}\right] \zeta+\frac{B k_{\mathrm{UE}}-k_{\mathrm{PCM}}}{\Delta k}
$$

By incorporating Eq. (A.2) into Eq. (A.1) with the same initial condition for Eq. (6) , the optimal enhancement ratio with $\lambda_{\mathrm{SE}}, \epsilon_{z, o p t}^{\prime}$, can be obtained. The dimensionless energy balance in 1-D cylindrical coordinates from Eq. (14) becomes

$$
\begin{aligned}
& -\left.\kappa(\rho) \frac{\partial \theta\left(\rho, \tau_{r}\right)}{\partial \rho}\right|_{\rho=\rho_{\mathrm{m}}\left(\tau_{r}\right)}= \\
& \left(\frac{1-v_{\mathrm{Mesh}}}{1-v_{\mathrm{Mesh}}^{\prime}}\right) \frac{\left(R_{\max }-1\right)^{2}}{\frac{R_{\max }^{2}}{2} \ln \left(R_{\max }\right)-\frac{R_{\max }^{2}}{4}+\frac{1}{4}} \frac{d \rho_{\mathrm{m}}\left(\tau_{r}\right)}{d \tau_{r}} .
\end{aligned}
$$

The volume fraction $v_{\text {Mesh }}^{\prime}$ using Eq. (20) takes the form

$$
\begin{aligned}
v_{\mathrm{Mesh}}^{\prime}(\rho)= & {\left[\left(\frac{3(1-H)\left(R_{\max }+1\right) R_{\min }^{2}}{2 R_{\max }^{3}-3 R_{\max }^{2}+1}\right) \frac{k_{\mathrm{UE}}}{\Delta k}\right] \rho } \\
& +\frac{H k_{\mathrm{UE}}-k_{\mathrm{PCM}}}{\Delta k} .
\end{aligned}
$$

By incorporating Eq. (A.4) into Eq. (A.3) with the same initial condition used for Eq. (14), the optimal enhancement ratio with $\lambda_{\mathrm{SE}}, \epsilon_{r, \mathrm{opt}}^{\prime}$, can be obtained. The relative difference is defined as

$$
\Delta \epsilon_{i, \mathrm{opt}}=\epsilon_{i, \mathrm{opt}}-\epsilon_{i, \mathrm{opt}}^{\prime}
$$

where the subscript $i=z$ or $r$.

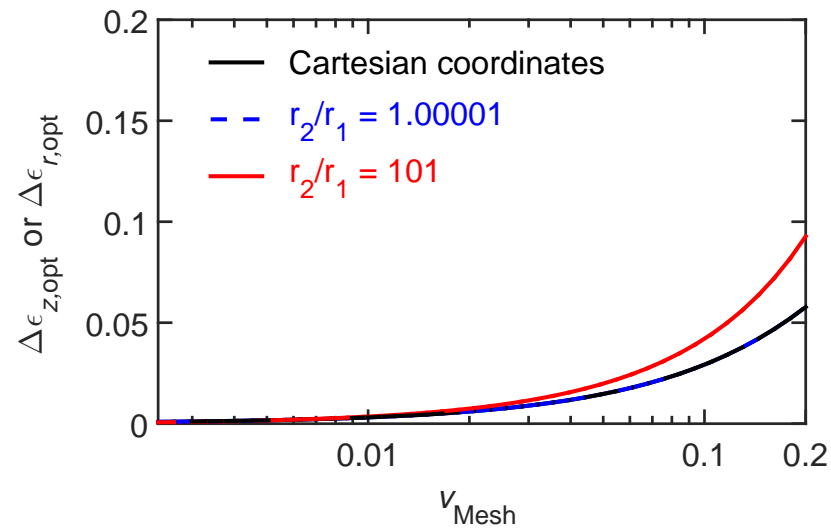

Figure A.1: The difference of the optimal enhancement ratio of the average charge/discharge using $\lambda$ and $\lambda_{\mathrm{SE}}$ versus $v_{\mathrm{Mesh}}$. With $v_{\text {Mesh }}<0.2$, the small difference indicates that the solutions using constant $\lambda$ are not significantly affected.

The relative difference of optimal enhancement ratio between using $\lambda$ and $\lambda_{\mathrm{SE}}$ from $v_{\mathrm{Mesh}}=0.001$ to $v_{\mathrm{Mesh}}=0.2$ is shown in Fig. A.1. The maximum difference reaches 0.1 with $v_{\text {Mesh }}=0.2$ and $r_{2} / r_{1}=101$. This difference implies that the change from $\lambda$ to $\lambda_{\mathrm{SE}}$ does not significantly decrease the optimal enhancement. This result validates the assumption of using the constant $\lambda$ to simplify our analysis. 


\section{Appendix B. Derivation of the maximum melting}

$$
\text { times }\left(t_{z, \max } \text { and } t_{r, \max }\right)
$$

The maximum melting time is the time for the melting front to reach $z_{\mathrm{m}}=d\left(t=t_{z \text {, max }}\right)$ in 1-D Cartesian coordinates or $r_{\mathrm{m}}=r_{2}\left(t=t_{r, \max }\right)$ in 1-D cylindrical coordinates. The maximum melting time for uniform mesh $\left(k_{\mathrm{UE}}\right)$ is determined when $k_{\mathrm{UE}}$ is substituted for $k(z)$ in Eq. (1) and Eq. (2) or for $k(r)$ in Eq. (9) and Eq. (10). The temporal temperature distribution $T(z, t)=T_{\mathrm{s}}+\left(T_{\mathrm{m}}-T_{\mathrm{s}}\right) z / z_{\mathrm{m}}$ in 1-D Cartesian coordinates is substituted into Eq. (2), and $t_{z, \max }$ is acquired by integration as

$$
\int_{0}^{t_{z, \max }} d t^{\prime}=\int_{0}^{z_{\mathrm{m}}=d} d z_{\mathrm{m}}^{\prime} z_{\mathrm{m}}^{\prime}\left[\frac{\lambda}{k_{\mathrm{UE}}\left(T_{\mathrm{s}}-T_{\mathrm{m}}\right)}\right]
$$

For 1-D cylindrical coordinates, $T(r, t)=T_{\mathrm{s}}+$ $\left(T_{\mathrm{m}}-T_{\mathrm{s}}\right) \ln \left(r / r_{1}\right) / \ln \left(r_{\mathrm{m}} / r_{1}\right)$ is substituted into Eq. (10), and $t_{r, \max }$ is acquired by integration as

$$
\begin{aligned}
& \int_{0}^{t_{r, \max }} d t^{\prime}= \\
& \int_{r_{1}}^{r_{\mathrm{m}}=r_{2}} d r_{\mathrm{m}}^{\prime} r_{\mathrm{m}}^{\prime} \ln \left(\frac{r_{\mathrm{m}}^{\prime}}{r_{1}}\right)\left[\frac{\lambda}{k_{\mathrm{UE}}\left(T_{\mathrm{s}}-T_{\mathrm{m}}\right)}\right]
\end{aligned}
$$

\section{Appendix C. Derivation of the enhancement ratio}

\section{in 1-D cylindrical coordinates $\left(\epsilon_{r}\right)$}

The enhancement ratio of the average charge/discharge rate is defined as the reciprocal of the nondimensionlized time with $\rho_{\mathrm{m}}=1$ and $R_{\mathrm{m}}=R_{\max }$ in Eq. (15), which is acquired by integration of Eq. (14).

We obtain Eq. (14) by substituting $d t=d \tau_{r} t_{r, \max }$, $k(r)=\kappa(\rho) k_{\mathrm{UE}}, d T(r, t)=d \theta\left(\rho, \tau_{r}\right)\left(T_{\mathrm{s}}-T_{\mathrm{m}}\right), d r=$ $d \rho\left(R_{\max }-1\right) r_{1}, \quad$ and $d r_{\mathrm{m}}=d \rho_{\mathrm{m}}\left(R_{\max }-1\right) r_{1} \quad$ into Eq. (10). After rearrangement, the right-hand-side of Eq. (14) takes the form

$$
\begin{aligned}
& -\left.\kappa(\rho) \frac{\partial \theta\left(\rho, \tau_{r}\right)}{\partial \rho}\right|_{\rho=\rho_{\mathrm{m}}\left(\tau_{r}\right)} \\
& =\left[\frac{\lambda\left(R_{\max }-1\right)^{2} r_{1}^{2}}{k_{\mathrm{UE}}\left(T_{\mathrm{s}}-T_{\mathrm{m}}\right) t_{r, \max }}\right] \frac{d \rho_{\mathrm{m}}\left(\tau_{r}\right)}{d \tau_{r}} \\
& =\left[\frac{\left(R_{\max }-1\right)^{2}}{\left.\frac{R_{\max }^{2} \ln \left(R_{\max }\right)-\frac{R_{\max }^{2}}{4}+\frac{1}{4}}{2}\right] \frac{d \rho_{\mathrm{m}}\left(\tau_{r}\right)}{d \tau_{r}} .}\right.
\end{aligned}
$$

Substituting the derivative of $\theta\left(\rho, \tau_{r}\right)$ from Eq. (13) into the left-hand side of Eq. (C.1) results in

$$
\begin{aligned}
& \int_{0}^{\tau_{r}} d \tau_{r}^{\prime}= \\
& \frac{R_{\min }^{2}}{\left(G R_{\min }-H\right)\left[\frac{R_{\max }^{2}}{2} \ln \left(R_{\max }\right)-\frac{R_{\max }^{2}}{4}+\frac{1}{4}\right]} \\
& \times\left\{\int_{0}^{\rho_{\mathrm{m}}} d \rho_{\mathrm{m}}^{\prime}\left(1+\rho_{\mathrm{m}}^{\prime} R_{\min }\right) \times\right. \\
& \left.\left[\ln \left(1+\rho_{\mathrm{m}}^{\prime} R_{\min }\right)-\ln \left(\frac{G}{H} \rho_{\mathrm{m}}^{\prime}+1\right)\right]\right\} .
\end{aligned}
$$

Then, the reciprocal of $\tau_{r}$ in Eq. (C.2) with $\rho_{\mathrm{m}}=1$ and $R_{\mathrm{m}}=R_{\max }$ is defined as the enhancement ratio in Eq. (16). Under the conservation of average volume fraction of mesh, we eliminate $G$ and work $\kappa(\rho)$ as a function of $H$ and $R_{\max }$ (Eq. (20)) to obtain the enhancement ratio as a function of $H$ and $R_{\max }$ in Eq. (21).

\section{Acknowledgements}

We are thankful for helpful discussions with Dr. WeeLiat Ong (Carnegie Mellon University). We acknowledge partial support from the Department of Energy via grant DE-EE0006702.

\section{References}

[1] S. Jegadheeswaran, S. D. Pohekar, Performance enhancement in latent heat thermal storage system: A review, Renewable and Sustainable Energy Reviews 13 (9) (2009) 2225-2244. 
[2] Y. Dutil, D. R. Rousse, N. B. Salah, S. Lassue, L. Zalewski, A review on phase-change materials: Mathematical modeling and simulations, Renewable and Sustainable Energy Reviews 15 (1) (2011) 112-130.

[3] J. Pereira da Cunha, P. Eames, Thermal energy storage for low and medium temperature applications using phase change materials a review, Applied Energy 177 (2016) 227-238.

[4] G. Ferrer, A. Sol, C. Barreneche, I. Martorell, L. F. Cabeza, Review on the methodology used in thermal stability characterization of phase change materials, Renewable and Sustainable Energy Reviews 50 (2015) 665-685.

[5] K. O. Lee, M. A. Medina, E. Raith, X. Sun, Assessing the integration of a thin phase change material ( $\mathrm{pcm}$ ) layer in a residential building wall for heat transfer reduction and management, Applied Energy 137 (2015) 699-706.

[6] B. Xu, P. Li, C. Chan, E. Tumilowicz, General volume sizing strategy for thermal storage system using phase change material for concentrated solar thermal power plant, Applied Energy 140 (2015) 256-268.

[7] B. Zalba, J. M. Marn, L. F. Cabeza, H. Mehling, Review on thermal energy storage with phase change: materials, heat transfer analysis and applications, Applied Thermal Engineering 23 (3) (2003) 251-283.

[8] M. Kenisarin, K. Mahkamov, Solar energy storage using phase change materials, Renewable and Sustainable Energy Reviews 11 (9) (2007) 1913-1965.

[9] F. Agyenim, N. Hewitt, P. Eames, M. Smyth, A review of materials, heat transfer and phase change problem formulation for latent heat thermal energy storage systems (lhtess), Renewable and Sustainable Energy Reviews 14 (2) (2010) 615-628.

[10] P. Zhang, X. Xiao, Z. W. Ma, A review of the composite phase change materials: Fabrication, characterization, mathematical modeling and application to performance enhancement, Applied Energy 165 (2016) 472-510.

[11] M. Medrano, M. O. Yilmaz, M. Nogus, I. Martorell, J. Roca, L. F. Cabeza, Experimental evaluation of commercial heat exchangers for use as pcm thermal storage systems, Applied Energy 86 (10) (2009) 2047-2055.

[12] K. Biswas, J. Lu, P. Soroushian, S. Shrestha, Combined experimental and numerical evaluation of a prototype nano-pcm enhanced wallboard, Applied Energy 131 (2014) 517-529.

[13] H. Mehling, L. Cabeza, Heat and cold storage with PCM: An up to date introduction into basics and applications, Springer
Berlin Heidelberg, 2008.

[14] A. Elgafy, K. Lafdi, Carbon 43 (2005) 3067.

[15] H. Huang, C. H. Liu, Y. Wu, S. Fan, Aligned carbon nanotube composite films for thermal management, Advanced Materials 17 (13) (2005) 1652-1656.

[16] S. Kim, L. T. Drzal, High latent heat storage and high thermal conductive phase change materials using exfoliated graphite nanoplatelets, Solar Energy Materials and Solar Cells 93 (1) (2009) 136-142.

[17] F. Yavari, H. R. Fard, K. Pashayi, M. A. Rafiee, A. Zamiri, Z. Yu, R. Ozisik, T. Borca-Tasciuc, N. Koratkar, Enhanced thermal conductivity in a nanostructured phase change composite due to low concentration graphene additives, The Journal of Physical Chemistry C 115 (17) (2011) 8753-8758.

[18] S. N. Schiffres, S. Harish, S. Maruyama, J. Shiomi, J. A. Malen, Tunable electrical and thermal transport in ice-templated multilayer graphene nanocomposites through freezing rate control, ACS Nano.

[19] M. Xia, Y. Yuan, X. Zhao, X. Cao, Z. Tang, Cold storage condensation heat recovery system with a novel composite phase change material, Applied Energy 175 (2016) 259-268.

[20] M. A. Kibria, M. R. Anisur, M. H. Mahfuz, R. Saidur, I. H. S. C. Metselaar, A review on thermophysical properties of nanoparticle dispersed phase change materials, Energy Conversion and Management 95 (2015) 69-89.

[21] H. Ji, D. P. Sellan, M. T. Pettes, X. Kong, J. Ji, L. Shi, R. S. Ruoff, Enhanced thermal conductivity of phase change materials with ultrathin-graphite foams for thermal energy storage, Energy \& Environmental Science 7 (3) (2014) 1185-1192.

[22] G. E. I. Humphries, W. R., A design handbook for phase change thermal control and energy storage devices, NASA Technical paper 1074 (1977) 256.

[23] L. Fan, J. M. Khodadadi, Thermal conductivity enhancement of phase change materials for thermal energy storage: A review, Renewable and Sustainable Energy Reviews 15 (1) (2011) 2446.

[24] L. F. Cabeza, H. Mehling, S. Hiebler, F. Ziegler, Heat transfer enhancement in water when used as pcm in thermal energy storage, Applied Thermal Engineering 22 (10) (2002) 1141-1151.

[25] K. Lafdi, O. Mesalhy, S. Shaikh, Experimental study on the influence of foam porosity and pore size on the melting of phase change materials, Journal of Applied Physics 102 (8) (2007) 083549 . 
[26] H. Yin, X. Gao, J. Ding, Z. Zhang, Experimental research on heat transfer mechanism of heat sink with composite phase change materials, Energy Conversion and Management 49 (6) (2008) 1740-1746.

[27] H. Wang, F. Wang, Z. Li, Y. Tang, B. Yu, W. Yuan, Experimental investigation on the thermal performance of a heat sink filled with porous metal fiber sintered felt/paraffin composite phase change material, Applied Energy 176 (2016) 221-232.

[28] M. Schuetz, L. Glicksman, A basic study of heat transfer through foam insulation, Journal of Cellular Plastics 20 (2) (1984) 114-121.

[29] J. P. Kruth, G. Levy, F. Klocke, T. H. C. Childs, Consolidation phenomena in laser and powder-bed based layered manufacturing, CIRP Annals - Manufacturing Technology 56 (2) (2007) 730-759.

[30] L. E. Murr, S. M. Gaytan, D. A. Ramirez, E. Martinez, J. Hernandez, K. N. Amato, P. W. Shindo, F. R. Medina, R. B. Wicker, Metal fabrication by additive manufacturing using laser and electron beam melting technologies, Journal of Materials Science Technology 28 (1) (2012) 1-14.

[31] B. Sarler, Stefan's work on solid-liquid phase changes, Engineering Analysis with Boundary Elements 16 (2) (1995) 83-92.

[32] L. Jiji, Heat Conduction, Springer Berlin Heidelberg, 2009.

[33] D. Tarzia, Explicit and approximated solutions for heat and mass transfer problems with a moving interface, in: M. El-Amin (Ed.), Advanced Topics in Mass Transfer, InTech, 2011.

[34] J. Nicolin Douglas, E. C. da Silva Gisleine, M. Jorge Regina Maria, M. Jorge Luiz Mario, Numerical solution of a nonlinear diffusion model for soybean hydration with moving boundary, International Journal of Food Engineering 11 (5) (2015) 587-595.

[35] J. Hill, V. Hart, The stefan problem in nonlinear heat conduction, Zeitschrift fr angewandte Mathematik und Physik ZAMP 37 (2) (1986) 206-229.

[36] C. Rogers, On a class of moving boundary problems in nonlinear heat conduction: Application of a bäcklund transformation, International Journal of Non-Linear Mechanics 21 (4) (1986) 249-256.

[37] C. Rogers, P. Broadbridge, On a nonlinear moving boundary problem with heterogeneity: application of a reciprocal transformation, Zeitschrift fr angewandte Mathematik und Physik ZAMP 39 (1) (1988) 122-128.

[38] M. F. Natale, D. A. Tarzia, Explicit solutions to the one-phase stefan problem with temperature-dependent thermal conductivity and a convective term, International Journal of Engineering Science 41 (15) (2003) 1685-1698.

[39] D. A. T. Adriana C. Briozzo, Mara F. Natale, The stefan problem with temperature-dependent thermal conductivity and a convective term with a convective condition at the fixed face, COMMUNICATIONS ON doi:10.3934/cpaa.2010.9.1209 PURE AND APPLIED ANALYSIS 9 (5) (2010) 12.

[40] V. R. Voller, F. Falcini, Two exact solutions of a stefan problem with varying diffusivity, International Journal of Heat and Mass Transfer 58 (12) (2013) 80-85.

[41] Y. Zhou, L.-j. Xia, Exact solution for stefan problem with general power-type latent heat using kummer function, International Journal of Heat and Mass Transfer 84 (2015) 114-118.

[42] T. Bergman, F. Incropera, A. Lavine, Fundamentals of Heat and Mass Transfer, Wiley, 2011.

[43] J. Wang, J. K. Carson, M. F. North, D. J. Cleland, A new approach to modelling the effective thermal conductivity of heterogeneous materials, International Journal of Heat and Mass Transfer 49 (1718) (2006) 3075-3083. 
Uniformly Enhanced

Thermal Conductivity $K$

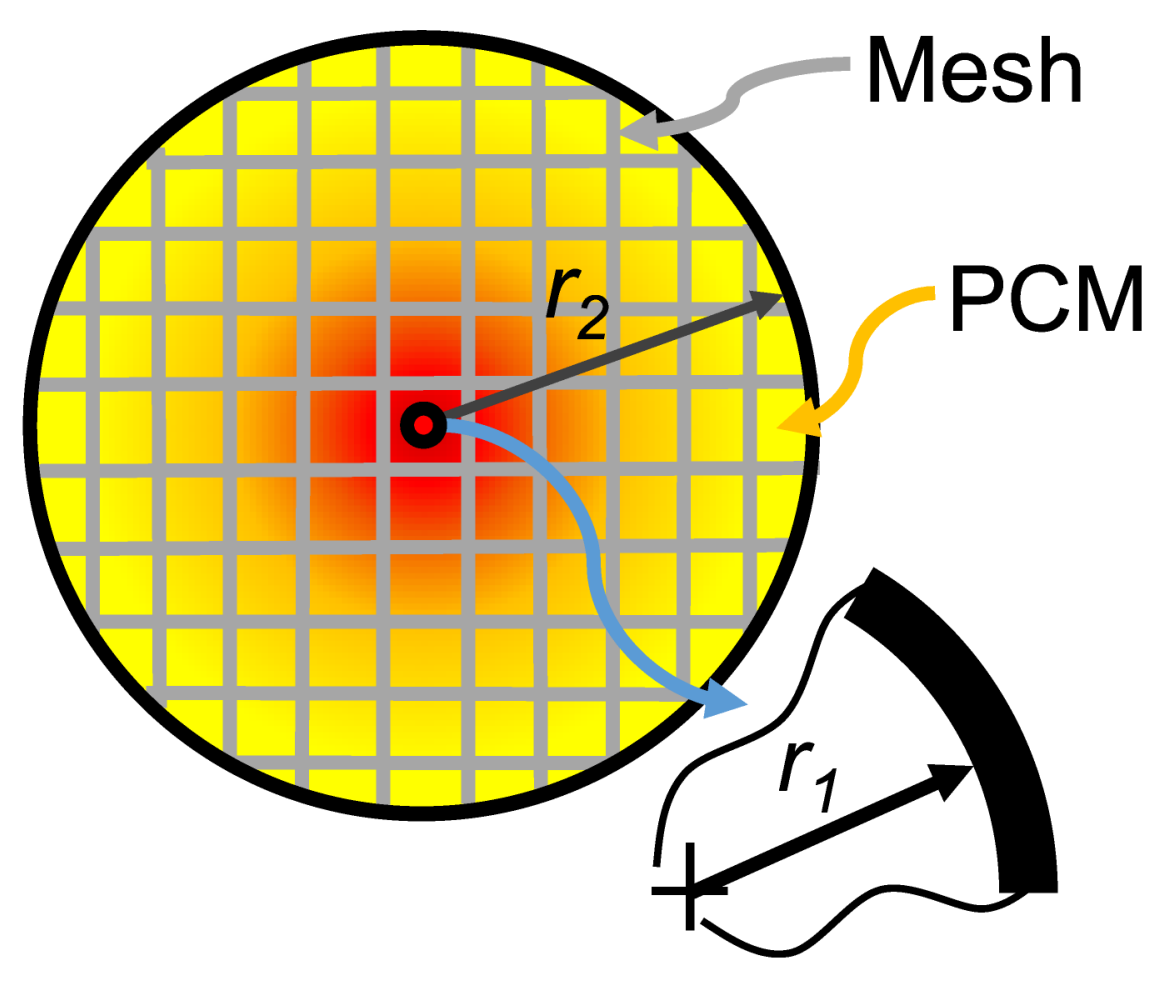

Spatially Dependent Thermal Conductivity $k$
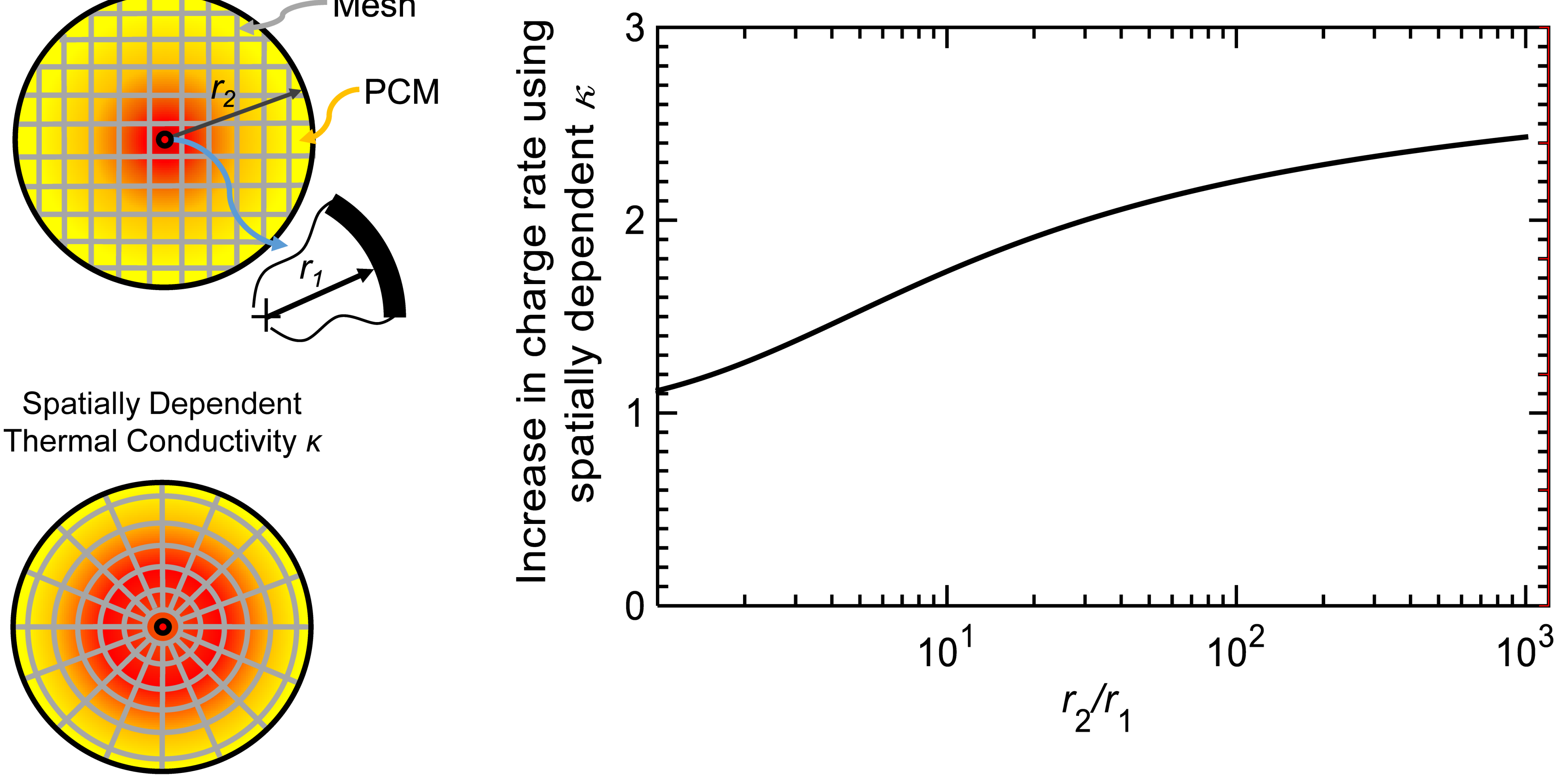